\title{
The Impact of the Location of Temperature Sensors on the Accuracy of Transient-State Temperature Distribution Identification
}

\author{
Mariusz Konieczny \\ Institute of Thermal and Process Engineering, Department of Mechanical Engineering, Cracow University of Technology \\ Al. Jana Pawła II 37, 31-864 Kraków, Poland \\ mk.mk@wp.pl
}

\section{Extended Abstract}

Many structures in various technical applications operate under strong thermal conditions. Steady- or transient-state heat transfer phenomena can create substantial temperature differences which should not exceed the allowable limit. The common problem of calculating the temperature field is the difficulty in accessing some of the thermal boundary conditions in operated elements. This usually concerns internal surfaces, where the fluid is in contact with the element. Numerical analysis of phenomena taking place in a flowing fluid is very time-consuming. Another way to determine the temperature distribution is to find the solution of the inverse heat conduction problem (IHCP) in the device under analysis [1-4] and verify it experimentally [5]. Despite the unknown boundary condition, the proposed method makes it possible to determine the temperature field using "measured" temperature histories determined in easily accessible points on the component outer surface, using energy balance equations. Unfortunately, the accuracy of the method is strongly dependent on the distance between the temperature sensors and the unknown boundary. The second factor creating the final error is the uncertainty of the thermocouple input data. For bigger distances, e.g. in a thick-walled pressure component burdened with measurement errors, oscillations appear in the solutions and the solution error can rise to unacceptable values.

Information about the error size is crucial in determining the potential use of specific applications. As a result of a series of transient-state numerical analyses, the final error value was determined as a function of the wall thickness and measurement inaccuracy. "Measurement" errors of $\pm 0.25, \pm 0.5$, and $\pm 1^{\circ} \mathrm{C}$ were assumed and implemented in the analysis as disturbance. Numerical tests were conducted for two types of a thick-walled pipe with the inner diameter of $160 \mathrm{~mm}$ and the wall thickness values of 40 and $60 \mathrm{~mm}$, respectively. It is assumed that the pipe with a uniform initial temperature distribution is partially flooded by the hot medium, which simulates the system heating process. The outer surface is exposed to ambient air. Additionally, in order to overcome instabilities, the influence of smoothing digital filters was investigated.

It may be stated that the expected standard deviation $\sigma<2$ (relative error measure $\mathrm{RE}<0.5 \%$ ) of the final result can be achieved for the input error of $\pm 0.5^{\circ} \mathrm{C}$ for the 40 -mm-thick wall, but the input data acceptable error for the wall thickness of $60 \mathrm{~mm}$ should be $\pm 0.25^{\circ} \mathrm{C}$ and smoothing filters must be used additionally. Both levels of temperature measurement accuracy are achievable for industrial thermocouples. For the wall thickness of $60 \mathrm{~mm}$ and the input error of $\pm 0.5^{\circ} \mathrm{C}$ (no filters applied), the standard deviation value is much higher: $\sigma \approx 5.1$ ( $\mathrm{RE} \approx 36 \%$ ). The algorithm is stable even for thick walls. However, for thicknesses over $50 \mathrm{~mm}$, it becomes more sensitive to input errors and requires additional filters to ensure stability.

\section{References}

[1] O.M. Alifanov, A.V. Nenarokomov, "Three-dimensional boundary inverse heat conduction problem for regular coordinate systems", Inverse Problems in Engineering, vol. 7, no. 4, pp. 335-362, 1999.

[2] M. Cui, Y. Zhao, B. Xu, X. Gao, "A new approach for determining damping factors in Levenberg-Marquardt algorithm for solving an inverse heat conduction problem", International Journal of Heat and Mass Transfer, 107 (2017) 747-754.

[3] P. Duda, "Solution of an inverse axisymmetric heat conduction problem in complicated geometry", Int. J. Heat Mass Transf., vol. 82, pp. 419-428, 2015. 
[4] P. Duda, M. Konieczny, "Solution of an inverse transient heat conduction problem in a part of a complex domain", Int. J. Heat Mass Transf., vol. 127, pp. 821-832, 2018.

[5] P. Duda, M. Konieczny, "Experimental verification of the inverse method of the heat transfer coefficient calculation", Energies, 13(6), 1440, 2020. 